\title{
The Impact of Using Genotyped Reagent Red Blood Cells in Antibody Identification
}

\author{
Erwin Scharberg ${ }^{a} \quad$ Gabi Rink $^{b} \quad$ Jan Portegys ${ }^{b}$ Sina Rothenberger ${ }^{a} \quad$ Nicole Gillhuber $^{a}$ \\ Ekkehard Richter ${ }^{\mathrm{a}}$ Peter Bugert ${ }^{\mathrm{b}}$ \\ ${ }^{a}$ Institute of Transfusion Medicine and Immunohematology, German Red Cross Blood Service Baden-Württemberg - Hesse, \\ Baden-Baden, Germany; \\ b Institute of Transfusion Medicine and Immunology, Heidelberg University, Medical Faculty Mannheim, German Red Cross \\ Blood Service Baden-Württemberg - Hessen, Mannheim, Germany
}

\section{Keywords}

Pre-transfusion diagnostics - Molecular blood typing · Antibody identification

\section{Summary \\ Background: The detection and identification of antibod- ies to red blood cell (RBC) antigens is one of the most important and challenging issues in transfusion medi- cine. Up to date there are $354 \mathrm{RBC}$ antigens recognized by the International Society of Blood Transfusion (ISBT). The reagent RBCs used in commercial antibody screen- ing and identification panels however are usually sero- logically typed for up to 40 clinically important antigens. Thus the identification of many antibody specificities re- mains impossible when using reagent RBCs with only limited information about their antigens. To improve the pre-transfusion diagnostics, we developed antibody identification panels with reagent RBCs serologically typed for 26 antigens and additionally genotyped for 30 blood group alleles. Methods: The reagent RBCs in the panels were characterized serologically for the clinically most significant 'standard' antigens. The reagent RBC donors were additionally genotyped by using in-house PCR-SSP methods. The antibody identification was per- formed in the indirect antiglobulin test using untreated and papain-treated RBCs in the gel technique. Antibod- ies identified due to the genotype information were con- firmed by serology using appropriate reference RBCs. Results: Within a time period of 3 years and 8 months, 16,878 blood samples from 8,467 patients were tested in our reference laboratory. In total, 21 different antibodies}

from 10 different blood group systems could be identified in 126 patients (1.5\%) due to the genotype information obtained for the reagent RBCs. Antibodies to antigens from the Knops system (53 patients; 42\%, 8 patients with anti-Kn ${ }^{\mathrm{b}}$ ) and to Cartwright antigens (31 patients; $25 \%$ ) were the most frequent. Conclusion: The use of genotyped reagent RBCs in antibody identification panels extends the range of detectable antibody specificities, accelerates the antibody identification, and improves the pre-transfusion diagnostics.

(c) 2018 S. Karger GmbH, Freiburg

\section{Introduction}

Since 1900 more than 350 red blood cell (RBC) antigens have been discovered. They are defined serologically by the use of a specific antibody. Antigens receiving ISBT numbers (assigned by the ISBT Working Party on Red Cell Immunogenetics and Blood Group Nomenclature) must have been shown to be inherited characters $[1,2]$. All antigens fall into one of the following classifications: systems, collections (200 series), low-incidence antigens (700 series), and high-incidence antigens (901 series). A blood group system consists of one or more antigens controlled at a single gene locus, or by two or more very closely linked homologous genes with little or no observable recombination between them (table 1). Collections consist of serologically, biochemically, or genetically related antigens, which do not fit the criteria required for system

\section{KARGER}

() 2018 S. Karger GmbH, Freiburg

Fax +497614520714
Prof. Dr. Erwin A. Scharberg

Institute of Transfusion Medicine and Immunohematology

German Red Cross Blood Service Baden-Württemberg - Hessen gGmbH

Gunzenbachstraße 35, 76530 Baden-Baden, Germany

a.scharberg@blutspende.de 
status (table 2). The 700 series contains antigens with an incidence of less than $1 \%$ and which cannot be included in a system or collection. The 901 series contains antigens with an incidence of greater than $90 \%$ and which cannot be included in a system or collection (table 2).

The most important parts of the pre-transfusion diagnostics are the $\mathrm{ABO}$ typing and the screening for clinically significant irregular (unexpected) antibodies in blood recipients. The national Guidelines in Germany ('Richtlinie zur Gewinnung von Blut und Blutbestandteilen und zur Anwendung von Blutprodukten') recommend reagent $\mathrm{RBC}$ phenotyping for at least 19 antigens $\left(\mathrm{C}, \mathrm{C}^{\mathrm{w}}, \mathrm{c}, \mathrm{D}, \mathrm{E}, \mathrm{e}\right.$, $\left.\mathrm{K}, \mathrm{k}, \mathrm{Fy}(\mathrm{a} / \mathrm{b}), \mathrm{Jk}(\mathrm{a} / \mathrm{b}), \mathrm{S}, \mathrm{s}, \mathrm{M}, \mathrm{N}, \mathrm{P} 1, \mathrm{Le}^{\mathrm{a}}, \mathrm{Le}^{\mathrm{b}}\right)$. The following antigens of the panels should be homozygous: $\mathrm{C}, \mathrm{c}, \mathrm{Fy}(\mathrm{a} / \mathrm{b}), \mathrm{Jk}(\mathrm{a} / \mathrm{b}), \mathrm{S}$, s. Reagent RBCs used in commercial antibody screening and identification panels are serologically typed for up to 40 clinically important antigens.

For most of the 354 known antigens (e.g. Knops, $\mathrm{Do}^{\mathrm{a} / \mathrm{b}}, \mathrm{Yt}^{\mathrm{a} / \mathrm{b}}$, Lan, $\mathrm{Jr}^{\mathrm{a}}, \mathrm{Au}^{\mathrm{a} / \mathrm{b}}$, etc.), there are no available licensed test sera because of source limitations. Thus the identification of many antibody specificities remains impossible when using reagent RBCs with only limited information about their antigens.

The knowledge of the genetic background of a blood group antigen is the prerequisite for the development and use of genotyping methods [3]. Nowadays almost every blood group antigen can be typed by analysis of a corresponding DNA marker. Most blood group antigens differ from their antithetic partner by the alteration of a single nucleotide in the DNA sequence of the blood group gene. By genotyping of these single nucleotide polymorphisms (SNPs), the blood group phenotype can reliably be derived. Usually, genotyping methods are based on the polymerase chain reaction (PCR) with the use of genomic DNA. The large variety of techniques that were developed during the last two decades differs generally in the type of chemistry for specific detection of the SNP alleles [4-7]. Among the first methods that were developed, the PCR with sequence-specific primers (PCR-SSP) was the most common. PCR-SSP is a very cost-effective and robust method especially for the analysis of a limited number of SNPs in single or a low number of samples.

This progress in molecular immunohematology significantly contributes to optimized blood supply of patients and to prevention of immunization to blood cell antigens [8]. To improve the pre-transfusion diagnostics we developed antibody identification panels with reagent RBCs serologically typed for 26 'standard' antigens and additionally genotyped for 30 blood group alleles. We demonstrate that the use of these panels in routine testing significantly improves the pre-transfusion diagnostics, extending the range of detectable antibody specificities.

Table 1. Blood group systems

\begin{tabular}{|c|c|c|}
\hline System & $\begin{array}{l}\text { Number of } \\
\text { antigens }\end{array}$ & Antigens \\
\hline $001 \mathrm{ABO}$ & 4 & $\mathrm{~A}, \mathrm{~B}, \mathrm{AB}, \mathrm{A} 1$ \\
\hline 002 MNS & 49 & $\begin{array}{l}\mathrm{M}, \mathrm{N}, \mathrm{S}, \mathrm{s}, \mathrm{U}, \mathrm{He}, \mathrm{Mi}^{\mathrm{a}}, \mathrm{M}^{\mathrm{c}}, \mathrm{Vw}, \mathrm{Mur}, \mathrm{M}^{\mathrm{g}}, \mathrm{Vr}, \mathrm{M}^{\mathrm{e}}, \mathrm{Mt}^{\mathrm{a}}, \mathrm{St}^{\mathrm{a}}, \mathrm{Ri}^{\mathrm{a}}, \mathrm{Cla}, \mathrm{Ny}^{\mathrm{a}}, \mathrm{Hil}, \mathrm{M}^{\mathrm{v}}, \mathrm{Far}, \mathrm{S}^{\mathrm{D}}, \mathrm{Mit}, \mathrm{Dantu}, \mathrm{Hop}, \mathrm{Nob} \\
\mathrm{En}^{\mathrm{a}}, \mathrm{En}{ }^{\mathrm{a}} \mathrm{KT}, \mathrm{N}^{\prime} \text { ', Or, DANE, TSEN, MINY, MUT, SAT, ERIK, Os }{ }^{\mathrm{a}} \text {, ENEP, ENEH, HAG, ENAV, MARS, ENDA, } \\
\text { ENEV, MNTD, SARA, KIPP, JENU }\end{array}$ \\
\hline $003 \mathrm{P} 1 \mathrm{Pk}$ & 3 & $\mathrm{P} 1, \mathrm{P}^{\mathrm{k}}, \mathrm{NOR}$ \\
\hline $004 \mathrm{RH}$ & 55 & $\begin{array}{l}\text { D, C, E, c, e, f, Ce, C' }{ }^{w}, C^{\mathrm{x}}, \mathrm{V}, \mathrm{E}^{\mathrm{w}}, \mathrm{G}, \mathrm{Hr}_{0}, \mathrm{Hr}, \mathrm{hr}^{\mathrm{s}}, \mathrm{VS}, \mathrm{C}^{\mathrm{G}}, \mathrm{CE}, \mathrm{D}^{\mathrm{w}}, \mathrm{c}-\mathrm{like}, \mathrm{cE}, \mathrm{hr} \mathrm{H}^{\mathrm{H}}, \mathrm{Rh} 29, \mathrm{Go}^{\mathrm{a}}, \mathrm{hr}^{\mathrm{B}}, \mathrm{Rh} 32, \mathrm{Rh} 33, \mathrm{Hr}^{\mathrm{B}} \text {, } \\
\text { Rh35, Be } \mathrm{a}^{\mathrm{a}} \text {, Evans, Rh39, Tar, Rh41, Rh42, Crawford, Nou, Riv, Sec, Dav, JAL, STEM, FPTT, MAR, BARC, JAHK, } \\
\text { DAK, LORC, CENR, CEST, CELO, CEAG, PARG, CEVF, CEWA }\end{array}$ \\
\hline $005 \mathrm{LU}$ & 24 & $\mathrm{Lu}^{\mathrm{a}}, \mathrm{Lu}^{\mathrm{b}}, \mathrm{Lu} 3, \mathrm{Lu} 4, \mathrm{Lu} 5, \mathrm{Lu} 6, \mathrm{Lu} 7, \mathrm{Lu} 8, \mathrm{Lu} 9, \mathrm{Lu} 11, \mathrm{Lu} 12, \mathrm{Lu} 13, \mathrm{Lu} 14, \mathrm{Lu} 16, \mathrm{Lu} 17, \mathrm{Au}^{\mathrm{a}}, \mathrm{Au}^{\mathrm{b}}, \mathrm{Lu} 20, \mathrm{Lu} 21, \mathrm{LURC}$ \\
\hline $006 \mathrm{KEL}$ & 36 & 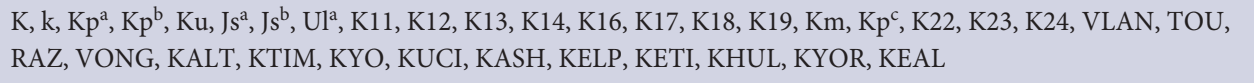 \\
\hline $007 \mathrm{LE}$ & 6 & $\mathrm{Le}^{\mathrm{a}}, \mathrm{Le}^{\mathrm{b}}, \mathrm{Le}^{\mathrm{ab}}, \mathrm{Le}^{\mathrm{bH}}, \mathrm{ALe}^{\mathrm{b}}, \mathrm{BLe}^{\mathrm{b}}$ \\
\hline $008 \mathrm{FY}$ & 5 & $\mathrm{Fy}^{\mathrm{a}}, \mathrm{Fy}^{\mathrm{b}}, \mathrm{Fy} 3, \mathrm{Fy} 5, \mathrm{Fy} 6$ \\
\hline $009 \mathrm{JK}$ & 3 & $\mathrm{Jk}^{\mathrm{a}}, \mathrm{Jk}^{\mathrm{b}}, \mathrm{Jk} 3$ \\
\hline $010 \mathrm{DI}$ & 22 & $\begin{array}{l}\mathrm{Di}^{\mathrm{a}}, \mathrm{Di}^{\mathrm{b}}, \mathrm{Wr}^{\mathrm{a}}, \mathrm{Wr}^{\mathrm{b}}, \mathrm{Wd}^{\mathrm{a}}, \mathrm{Rb}^{\mathrm{a}}, \mathrm{WARR}, \mathrm{ELO}, \mathrm{Wu}, \mathrm{Bp}^{\mathrm{a}}, \mathrm{Mo}^{\mathrm{a}}, \mathrm{Hg}^{\mathrm{a}}, \mathrm{Vg}^{\mathrm{a}}, \mathrm{Sw}^{\mathrm{a}}, \mathrm{BOW}, \mathrm{NFLD}, \mathrm{Jn}^{\mathrm{a}}, \mathrm{KREP}, \mathrm{Tr}^{\mathrm{a}}, \mathrm{Fr}^{\mathrm{a}}, \mathrm{SW} 1 \text {, } \\
\text { DISK }\end{array}$ \\
\hline $011 \mathrm{YT}$ & 3 & $\mathrm{Yt}^{\mathrm{a}}, \mathrm{Yt}^{\mathrm{b}}, \mathrm{YTEG}$ \\
\hline $012 \mathrm{XG}$ & 2 & $\mathrm{Xg}^{\mathrm{a}}, \mathrm{CD} 99$ \\
\hline 013 SC & 7 & Sc1, Sc2, Sc3, Rd, STAR, SCER, SCAN \\
\hline 014 DO & 10 & Do $^{\mathrm{a}}, \mathrm{Do}^{\mathrm{b}}, \mathrm{Gy}^{\mathrm{a}}, \mathrm{Hy}, \mathrm{Jo}^{\mathrm{a}}$, DOYA, DOMR, DOLG, DOLC, DODE \\
\hline $015 \mathrm{CO}$ & 4 & $\mathrm{Co}^{\mathrm{a}}, \mathrm{Co}^{\mathrm{b}}, \mathrm{Co} 3, \mathrm{Co} 4$ \\
\hline 016 LW & 3 & $\mathrm{LW}^{\mathrm{a}}, \mathrm{LW}^{\mathrm{b}}, \mathrm{LW}^{\mathrm{ab}}$ \\
\hline $017 \mathrm{CH} / \mathrm{RG}$ & 9 & Ch1, Ch2, Ch3, Ch4, Ch5, Ch6, WH, Rg1, Rg2 \\
\hline $018 \mathrm{H}$ & 1 & $\mathrm{H}$ \\
\hline 019 XK & 1 & $\mathrm{Kx}$ \\
\hline $020 \mathrm{GE}$ & 11 & Ge2, Ge3, Ge4, Wb, Ls ${ }^{\mathrm{a}}, \mathrm{An}^{\mathrm{a}}, \mathrm{Dh}^{\mathrm{a}}$, GEIS, GELP, GEAT, GETI \\
\hline
\end{tabular}


Table 1. Continued

\begin{tabular}{|c|c|c|}
\hline System & $\begin{array}{l}\text { Number of } \\
\text { antigens }\end{array}$ & Antigens \\
\hline $021 \mathrm{CROM}$ & 19 & $\begin{array}{l}\mathrm{Cr}^{\mathrm{a}}, \mathrm{Tc}^{\mathrm{a}}, \mathrm{Tc}^{\mathrm{b}}, \mathrm{Tc}^{\mathrm{c}}, \mathrm{Dr}^{\mathrm{a}}, \mathrm{Es}^{\mathrm{a}}, \mathrm{IFC}, \mathrm{WES}^{\mathrm{a}}, \mathrm{WES}^{\mathrm{b}}, \mathrm{UMC}, \mathrm{GUTI}, \mathrm{SERF}, \mathrm{ZENA}, \mathrm{CROV}, \mathrm{CRAM}, \mathrm{CROZ}, \mathrm{CRUE}, \mathrm{CRAG} \text {, } \\
\text { CROK }\end{array}$ \\
\hline $022 \mathrm{KN}$ & 9 & $\mathrm{Kn}^{\mathrm{a}}, \mathrm{Kn}^{\mathrm{b}}, \mathrm{McC}^{\mathrm{a}}, \mathrm{Sl} 1, \mathrm{Yk}^{\mathrm{a}}, \mathrm{McC}^{\mathrm{b}}, \mathrm{Sl} 2, \mathrm{Sl} 3, \mathrm{KCAM}$ \\
\hline 023 IN & 5 & $\mathrm{In}^{\mathrm{a}}, \mathrm{In}^{\mathrm{b}}$, INFI, INJA, INRA \\
\hline $024 \mathrm{OK}$ & 3 & $\mathrm{Ok}^{\mathrm{a}}, \mathrm{OKGV}, \mathrm{OKVM}$ \\
\hline 025 RAPH & 1 & MER2 \\
\hline $026 \mathrm{JMH}$ & 6 & JMH, JMHK, JMHL, JMHG, JMHM, JMHQ \\
\hline $027 \mathrm{I}$ & 1 & I \\
\hline 028 GLOB & 2 & P, PX2 \\
\hline 029 GIL & 1 & GIL \\
\hline 030 RHAG & 4 & Duclos, $\mathrm{Ol}^{\mathrm{a}}$, DSLK, RHAG4 \\
\hline 031 FORS & 1 & FORS1 \\
\hline $032 \mathrm{JR}$ & 1 & $\mathrm{Jr}^{\mathrm{a}}$ \\
\hline 033 LAN & 1 & Lan \\
\hline $034 \mathrm{VEL}$ & 1 & Vel \\
\hline 035 CD59 & 1 & CD59 \\
\hline 036 AUG & 2 & AUG1, AUG2 \\
\hline
\end{tabular}

Table 2. Blood group collections, low and high incidence antigens of the 700 and 901 series

\begin{tabular}{|c|c|c|}
\hline No. & Name & Symbol \\
\hline \multicolumn{3}{|c|}{ Collection } \\
\hline 205 & COsT & $\mathrm{Cs}^{\mathrm{a}}, \mathrm{Cs}^{\mathrm{b}}$ \\
\hline 207 & I & $\mathrm{i}$ \\
\hline 208 & ER & $\mathrm{Er}^{\mathrm{a}}, \mathrm{Er}^{\mathrm{b}}, \mathrm{Er} 3$ \\
\hline 209 & GLOB & LKE \\
\hline 210 & & $\mathrm{Le}^{\mathrm{c}}, \mathrm{Le}^{\mathrm{d}}$ \\
\hline 213 & MNCHO & $\mathrm{Hu}, \mathrm{M}_{1}, \mathrm{Tm}, \mathrm{Can}, \mathrm{Sext}, \mathrm{Sj}$ \\
\hline \multicolumn{3}{|c|}{ Low-incidence antigens (700 series) } \\
\hline 700002 & Batty & By \\
\hline 700003 & Christiansen & $\mathrm{Chr}^{\mathrm{a}}$ \\
\hline 700005 & Biles & $\mathrm{Bi}$ \\
\hline 700006 & Box & $\mathrm{Bx}^{\mathrm{a}}$ \\
\hline 700017 & Torkildsen & $\mathrm{To}^{\mathrm{a}}$ \\
\hline 700018 & Peters & $\mathrm{Pt}^{\mathrm{a}}$ \\
\hline 700019 & Reid & $\operatorname{Re}^{\mathrm{a}}$ \\
\hline 700021 & Jensen & $\mathrm{Je}^{\mathrm{a}}$ \\
\hline 700028 & Livesay & $\mathrm{Li}^{\mathrm{a}}$ \\
\hline 700039 & Milne & \\
\hline 700040 & Rasmussen & RASM \\
\hline 700044 & & JFV \\
\hline 700045 & Katagiri & $\mathrm{Kg}$ \\
\hline 700047 & Jones & JONES \\
\hline 700049 & & HJK \\
\hline 700050 & & HOFM \\
\hline 700054 & & REIT \\
\hline \multicolumn{3}{|c|}{ High-incidence antigens (901 series) } \\
\hline 901008 & & Emm \\
\hline 901009 & Anton & $\mathrm{AnWj}$ \\
\hline 901012 & Sid & $\mathrm{Sd}^{\mathrm{a}}$ \\
\hline 901014 & & PEL \\
\hline 901015 & & ABTI \\
\hline 901016 & & MAM \\
\hline
\end{tabular}

\section{Material and Methods}

Test Cell Panels

The reagent RBCs in the panels were tested serologically with licensed test sera for the following clinically most significant 'standard' antigens: RhD, C, c, E, e, $\mathrm{C}^{\mathrm{w}}, \mathrm{K}, \mathrm{k}, \mathrm{Kp}^{\mathrm{a}} / \mathrm{Kp}^{\mathrm{b}}, \mathrm{Fy}^{\mathrm{a}} / \mathrm{Fy}^{\mathrm{b}}, \mathrm{Jk}^{\mathrm{a}} / \mathrm{Jk}^{\mathrm{b}}, \mathrm{Le}^{\mathrm{a}} / \mathrm{Le}^{\mathrm{b}}, \mathrm{Lu}^{\mathrm{a}} / \mathrm{Lu}^{\mathrm{b}} \mathrm{P} 1, \mathrm{M}, \mathrm{N}, \mathrm{S}, \mathrm{s}, \mathrm{Xg}^{\mathrm{a}}$. In addition, 30 alleles (table 3 ) were typed by using in-house PCR-SSP methods according to a previously published standard protocol (fig. 1) [9]. $\mathrm{C}^{\mathrm{w}}$-positive donors were genotyped for the $\mathrm{C}^{\mathrm{w}}$ allele ( $\mathrm{RHCE}^{\star} 02.08$ ) in order to identify $\mathrm{RHCE}^{*} 02.08$ homozygous individuals who are negative for the MAR (RH51) antigen [10]. For that purpose a specific TaqMan ${ }^{\mathrm{TM}}$ PCR assay with primers (forward: TTTTTACCCACTATGACGCTTCCTT; reverse: CTGTTCCAATGAACTCTCACCTTGA) and fluorescently labeled minor groove binding probes (for $\mathrm{C}^{\mathrm{w}}$ : FAM-CCCTTTCGATCCTC; for non- $\mathrm{C}^{\mathrm{w}}$ : VIC-CCCCTTTTGATCCTC) was used according to the standard protocol of the manufacturer (Life Technologies, Darmstadt, Germany). Among 1,506 donors who had been previously phenotyped $\mathrm{C}^{\mathrm{w}}$-positive on the blood group analyzer Backman Coulter PK7200 we identified 25 donors homozygous for the RHCE ${ }^{\star} 02.08$ allele.

The antibody screening and identification were performed in the indirect antiglobulin test using untreated and papain-treated RBCs in the gel technique (Gel cards of BioRad, Cressier, Switzerland; and Grifols, Barcelona, Spain). Antibodies identified due to the genotype information were confirmed by serology using appropriate reference RBCs.

\section{Results}

The genotyped reagent RBC panels were introduced for routine use in our reference laboratory in August 2014. Over a time period of 3 years and 8 months, 16,878 samples from 8,467 patients were analyzed. In 234 blood samples (1.4\%) derived from 126 patients (1.5\%), the genotype information obtained for the test cells led to the identification and specification of an antibody (table 4). Overall, 21 different antibody specificities against antigens from 10 different blood group systems were identified. Antibodies to antigens from the Knops system $(\mathrm{KN})$ were the most frequent (53 of 126 
Table 3. Blood group alleles determined for the reagent $\mathrm{RBCs}$

\begin{tabular}{|c|c|c|c|c|c|}
\hline System (ISBT No.) & $\begin{array}{l}\text { Antigen/ } \\
\text { antithetic }\end{array}$ & Gene & $\begin{array}{l}\text { Antigen coding } \\
\text { genetic marker* }\end{array}$ & $\begin{array}{l}\text { ISBT allele } \\
\text { number }\end{array}$ & $\begin{array}{l}\text { Number in } \\
\text { dbSNP }\end{array}$ \\
\hline $\mathrm{Rh}(004)$ & $\begin{array}{l}\mathrm{C}^{\mathrm{W}}(\mathrm{RH} 8) \\
\mathrm{C}^{\mathrm{X}}(\mathrm{RH} 9)\end{array}$ & RHCE & $\begin{array}{l}122 \mathrm{G}(41 \mathrm{Arg}) \\
106 \mathrm{~A}(36 \mathrm{Thr})\end{array}$ & $\begin{array}{l}\text { RHCE } 02.08 \\
\text { RHCE } 02.09\end{array}$ & $\begin{array}{l}\text { rs138268848 } \\
\text { rs145034271 }\end{array}$ \\
\hline Lutheran (005) & $\begin{array}{l}\text { LU8 } \\
\text { LU14 } \\
\mathrm{Au}(\mathrm{a}) \\
\mathrm{Au}(\mathrm{b})\end{array}$ & $\begin{array}{l}B C A M \\
B C A M\end{array}$ & $\begin{array}{l}\text { 655T (204Met) } \\
655 \mathrm{~A} \text { (204Lys) } \\
\text { 1615A (539Thr) } \\
\text { 1615G (539Ala) }\end{array}$ & $\begin{array}{l}L U^{*} 02 \\
L U^{*} 02.14 \\
L U^{*} 02 \\
L U^{*} 02.19\end{array}$ & $\begin{array}{l}\text { rs28399656 } \\
\text { rs1135062 }\end{array}$ \\
\hline Kell (006) & $\begin{array}{l}\text { Js(a) (KEL6) } \\
\text { Js(b) (KEL7) } \\
\text { Côté (KEL11) } \\
\text { Wk(a) (KEL17) }\end{array}$ & $\begin{array}{l}K E L \\
K E L\end{array}$ & $\begin{array}{l}\text { 2000C (597Pro) } \\
\text { 2000T (597Leu) } \\
\text { 905T (302 Val) } \\
905 \mathrm{C} \text { (302Ala) }\end{array}$ & $\begin{array}{l}K E L^{*} 02.06 \\
K E L^{*} 02 \\
K E L^{*} 02 \\
K E L^{*} 02.17\end{array}$ & $\begin{array}{l}\text { rs8176038 } \\
\text { rs61729034 }\end{array}$ \\
\hline Diego (010) & $\begin{array}{l}\operatorname{Di}(\mathrm{a})(\mathrm{DI} 1) \\
\operatorname{Di}(\mathrm{b})(\mathrm{DI} 2)\end{array}$ & SLC4A1 & $\begin{array}{l}2710 \mathrm{~T} \text { (854Leu) } \\
2710 \mathrm{C} \text { (854Pro) }\end{array}$ & $\begin{array}{l}D I^{*} 01 \\
D I^{*} 02\end{array}$ & rs2285644 \\
\hline Cartwright (011) & $\begin{array}{l}\text { Yt(a) (YT1) } \\
\text { Yt(b) (YT2) }\end{array}$ & ACHE & $\begin{array}{l}\text { 1196C (353His) } \\
\text { 1196A (353Asn) }\end{array}$ & $\begin{array}{l}Y T^{*} 01 \\
Y T^{*} 02\end{array}$ & rs1799805 \\
\hline Scianna (013) & $\begin{array}{l}\mathrm{Sc} 1(\mathrm{SC} 1) \\
\mathrm{Sc} 2(\mathrm{SC} 2)\end{array}$ & ERMAP & $\begin{array}{l}169 \mathrm{G}(57 \mathrm{Gly}) \\
169 \mathrm{~A}(57 \mathrm{Arg})\end{array}$ & $\begin{array}{l}S C^{*} 01 \\
S C^{*} 02\end{array}$ & rs56025238 \\
\hline Dombrock (014) & $\begin{array}{l}\text { Do(a) (DO1) } \\
\text { Do(b) (DO2) }\end{array}$ & ART4 & $\begin{array}{l}\text { 1159A (265Asn) } \\
1159 \mathrm{G}(265 \mathrm{Asp})\end{array}$ & $\begin{array}{l}D O^{*} 01 \\
D O^{*} 02\end{array}$ & rs11276 \\
\hline Landsteiner-Wiener (016) & $\begin{array}{l}\text { LW(a) (LW5) } \\
\text { LW(b) (LW7) }\end{array}$ & ICAM4 & $\begin{array}{l}\text { 299A (100Gln) } \\
\text { 299G (100Arg) }\end{array}$ & $\begin{array}{l}L W^{*} 05 \\
L W^{*} 07\end{array}$ & rs77493670 \\
\hline Knops (022) & $\begin{array}{l}\mathrm{Kn}(\mathrm{a})(\mathrm{KN} 1) \\
\mathrm{Kn}(\mathrm{b})(\mathrm{KN} 2)\end{array}$ & CR1 & $\begin{array}{l}4708 \mathrm{G} \text { (1561 Val) } \\
4708 \mathrm{~A} \text { (1561Met) }\end{array}$ & $\begin{array}{l}K N^{*} 01 \\
K N^{*} 02\end{array}$ & rs41274768 \\
\hline & $\begin{array}{l}\mathrm{McC}(\mathrm{a})(\mathrm{KN} 3) \\
\mathrm{McC}(\mathrm{b})(\mathrm{KN} 6)\end{array}$ & CR1 & $\begin{array}{l}\text { 4795A (1590Lys) } \\
4795 \mathrm{G} \text { (1590Glu) }\end{array}$ & $\begin{array}{l}K N^{*} 01 \\
K N^{*} 01.06\end{array}$ & rs 17047660 \\
\hline & $\begin{array}{l}\text { Sl(a) (KN4) } \\
\text { Vil (KN7) }\end{array}$ & CR1 & $\begin{array}{l}4828 \mathrm{~A}(1601 \mathrm{Arg}) \\
4828 \mathrm{G}(1601 \mathrm{Gly})\end{array}$ & $\begin{array}{l}K N^{*} 01 \\
K N^{\star} 01.07\end{array}$ & rs17047661 \\
\hline & $\begin{array}{l}\mathrm{Yk}(\mathrm{a}-)(\mathrm{KN}-05) \\
\text { KCAM- }(\mathrm{KN}-09)\end{array}$ & $\begin{array}{l}\text { CR1 } \\
\text { CR1 }\end{array}$ & $\begin{array}{l}4223 \mathrm{~T} \text { (1408Met) } \\
4843 \mathrm{G}(1615 \mathrm{Val})\end{array}$ & $\begin{array}{l}K N^{*} 01 .-05 \\
K N^{*} 01 .-09\end{array}$ & $\begin{array}{l}\text { rs3737002 } \\
\text { rs6691117 }\end{array}$ \\
\hline Indian (023) & $\begin{array}{l}\text { In(a) (IN1) } \\
\text { In(b) (IN2) }\end{array}$ & $I N$ & $\begin{array}{l}\text { 137C (46Pro) } \\
137 \mathrm{G}(46 \mathrm{Arg})\end{array}$ & $\begin{array}{l}I N^{*} 01 \\
I N^{*} 02\end{array}$ & rs369473842 \\
\hline VEL (034) & Vel- (VEL-01) & SMIM1 & 64-80del & $V E L^{*}-01$ & rs566629828 \\
\hline
\end{tabular}

Fig. 1. Representative results from PCR-SSP typing of donor samples for the test cell panels. Specificity of the PCR-SSP is given by the allele number above. Deduced antigens are listed on the right with rare phenotypes highlighted.

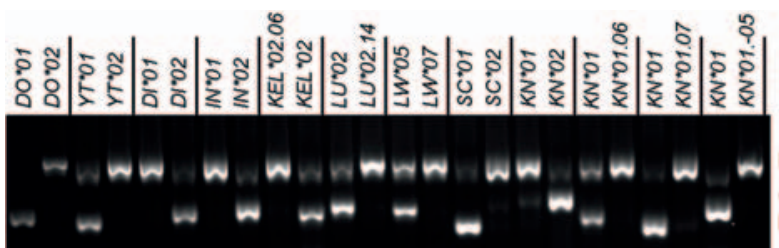

Do(a+b-), Yt(a+b-), Di(a-b+), $\ln (a-b+)$, Js $(a-b+), \operatorname{Lu}(8+14-), \operatorname{LW}(a+b-), \operatorname{Sc}(1+2-)$ Kn(a-be), McC(a+b-), SI(a+)Vil-, Yk(a+)

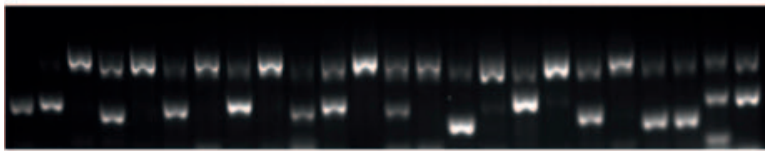

$D o(a+b+), Y i(-b+), D i(a-b+), \ln (a-b+)$, Js(a-b+), Lu(8+14-), LW(a+b-), Sc(1+2-), $\mathrm{Kn}(\mathrm{a}+\mathrm{b}-), \mathrm{McC}(\mathrm{a}+\mathrm{b}-), \mathrm{SI}\left(\mathrm{a}+\mathrm{VIII}^{+}, \mathrm{Yk}(\mathrm{a}+)\right.$

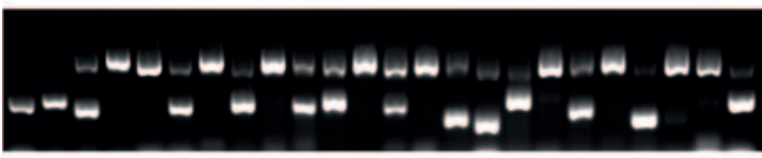

$D o(a+b+), Y t(a+b-), D i(a-b+), \ln (a-b+)$, $\mathrm{Js}(\mathrm{a}-\mathrm{b}+), \mathrm{Lu}(8+14-), \mathrm{LW}(\mathrm{a}+\mathrm{b}-), \mathrm{Sc}(1+2-)$, $\mathrm{Kn}(\mathrm{a}+\mathrm{b}-), \mathrm{McC}(\mathrm{a}+\mathrm{b}-), \mathrm{SI}(\mathrm{a}+) \mathrm{Vil}-$, nea-

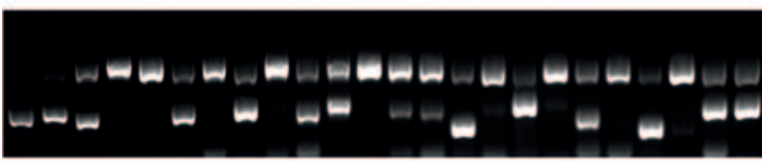

$\mathrm{Do}(\mathrm{a}+\mathrm{b}+), \mathrm{Yt}(\mathrm{a}+\mathrm{b}-), \mathrm{Di}(\mathrm{a}-\mathrm{b}+), \ln (\mathrm{a}-\mathrm{b}+)$ $\mathrm{Js}(\mathrm{a}-\mathrm{b}+), \mathrm{Lu}(8+14-), \mathrm{LW} \mathrm{a}+\mathrm{b}+), \mathrm{Sc}(1+2-)$ $\mathrm{Kn}(\mathrm{a}+\mathrm{b}-), \mathrm{McC}(\mathrm{a}+\mathrm{b}-), \mathrm{SI}(\mathrm{a}+) \mathrm{Vil}-, \mathrm{Yk}(\mathrm{a}+)$

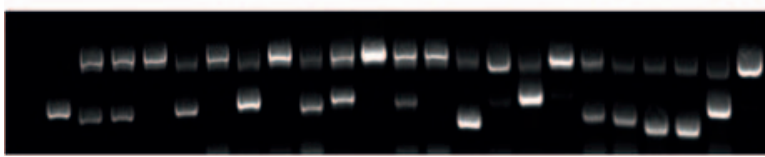

$\mathrm{Do}(\mathrm{a}-\mathrm{b}+), \mathrm{Yt}(\mathrm{a}+\mathrm{b}+), \mathrm{Di}(\mathrm{a}-\mathrm{b}+), \ln (\mathrm{a}-\mathrm{b}+)$, Js(a-b+), Lu(8+14-), LW(a+b-), Sc(1+2-), $\mathrm{Kn}(\mathrm{a}+\mathrm{b}-), \mathrm{MoCl}(\mathrm{a}+\mathrm{b}+), \mathrm{SI}(\mathrm{a}+) \mathrm{Vil}+, \mathrm{Yk}(\mathrm{a}+)$ 
Table 4. Antibodies identified in 8,467 patients ( 16,878 blood samples) based on the genotype information of the reagent RBCs

\begin{tabular}{|c|c|c|c|}
\hline System & $\begin{array}{l}\text { Antibody } \\
\text { specificity }\end{array}$ & $\begin{array}{l}\text { Number of } \\
\text { patients }\end{array}$ & $\begin{array}{l}\text { Number of } \\
\text { samples }\end{array}$ \\
\hline \multirow[t]{4}{*}{$\mathrm{KN}$} & $\mathrm{Kn}^{\mathrm{a}}$ & 20 & 29 \\
\hline & $\mathrm{Kn}^{\mathrm{b}}$ & 8 & 28 \\
\hline & $\mathrm{Yk}^{\mathrm{a}}$ & 19 & 28 \\
\hline & KCAM & 6 & 22 \\
\hline \multirow[t]{2}{*}{ YT } & $\mathrm{Yt}^{\mathrm{a}}$ & 19 & 29 \\
\hline & $\mathrm{Yt}^{\mathrm{b}}$ & 12 & 14 \\
\hline VEL & Vel & 7 & 9 \\
\hline \multirow[t]{2}{*}{ DO } & $\mathrm{Do}^{\mathrm{a}}$ & 5 & 6 \\
\hline & $\mathrm{Do}^{\mathrm{b}}$ & 2 & 2 \\
\hline \multirow[t]{3}{*}{ LU } & Lu14 & 3 & 18 \\
\hline & Lu8 & 3 & 7 \\
\hline & $A u^{b}$ & 3 & 3 \\
\hline \multirow[t]{2}{*}{$\mathrm{RH}$} & MAR & $4^{\star}$ & 4 \\
\hline & Tar & 1 & 2 \\
\hline \multirow[t]{2}{*}{ DI } & $\mathrm{Di}^{\mathrm{a}}$ & 2 & 21 \\
\hline & $\mathrm{Wu}$ & 1 & 1 \\
\hline $\mathrm{CO}$ & $\mathrm{Co}^{\mathrm{a}}$ & 4 & 4 \\
\hline \multirow[t]{2}{*}{ SC } & $\mathrm{Scl}$ & $2^{*}$ & 2 \\
\hline & Sc2 & 1 & 1 \\
\hline \multirow[t]{3}{*}{ LW } & $\mathrm{LW}^{\mathrm{a}}$ & 3 & 3 \\
\hline & $\mathrm{LW}^{\mathrm{b}}$ & 1 & 1 \\
\hline & Total & 126 & 234 \\
\hline
\end{tabular}

Fig. 2. Antibody identification using untreated and papaintreated reagent RBCs. Patient's plasma containing anti-Do ${ }^{\mathrm{b}}$ and anti-E. Both antibodies weakly reactive with untreated cells (most heterozygous cells not reactive). They are stronger reactive with papain treated cells in AHG. Red circles mark $\mathrm{Do}^{\mathrm{b}}$-negative and RhEnegative cells. patients; $42 \%$ ), followed by antibodies to the Cartwright antigens (31 of 126 patients; $25 \%$ ).

Some of the patients were repeatedly tested because of multiple transfusions or for control testing during a pregnancy. In all these cases the re-identification of the antibody was possible and could be performed in a short time. Three example cases (anti-Do ${ }^{\mathrm{a}}+$ anti-E, anti- $\mathrm{Au}^{\mathrm{b}}+$ anti-Lu ${ }^{\mathrm{a}}$, and anti-MAR) that were tested in our laboratory are demonstrated in figures $2-4$.

\section{Discussion}

To improve the pre-transfusion diagnostics, we developed antibody identification panels with reagent RBCs serologically typed for 26 'standard' antigens and additionally genotyped for 30 blood group alleles. The use of these panels led to the identification of antibody specificities difficult to obtain with standard commercial panels. In our patient population $1.5 \%$ of the cases contained such antibodies.

The most frequent specificities (42\%) were antibodies to the Knops antigens $\mathrm{Kn}^{\mathrm{a}}, \mathrm{Yk}^{\mathrm{a}}, \mathrm{Kn}^{\mathrm{b}}$ and KCAM. Unexpectedly we found anti- $\mathrm{Kn}^{\mathrm{b}}$ in 28 samples of 8 different patients. It had been reported only once in the literature [11] but now seems to be quite a common antibody. We also identified 6 patients with anti-KCAM which was reported in 2 cases only [12]. Interestingly, we found neither anti$\mathrm{McC}^{\mathrm{a}}$ nor anti- $\mathrm{McC}^{\mathrm{b}}$ in our patient population. Patients with Knops antibodies could receive transfusions without an increased risk of hemolytic transfusion reactions. Most of the identified Knops antibodies as well as the Dombrock, Cartwright, Scianna and Lutheran

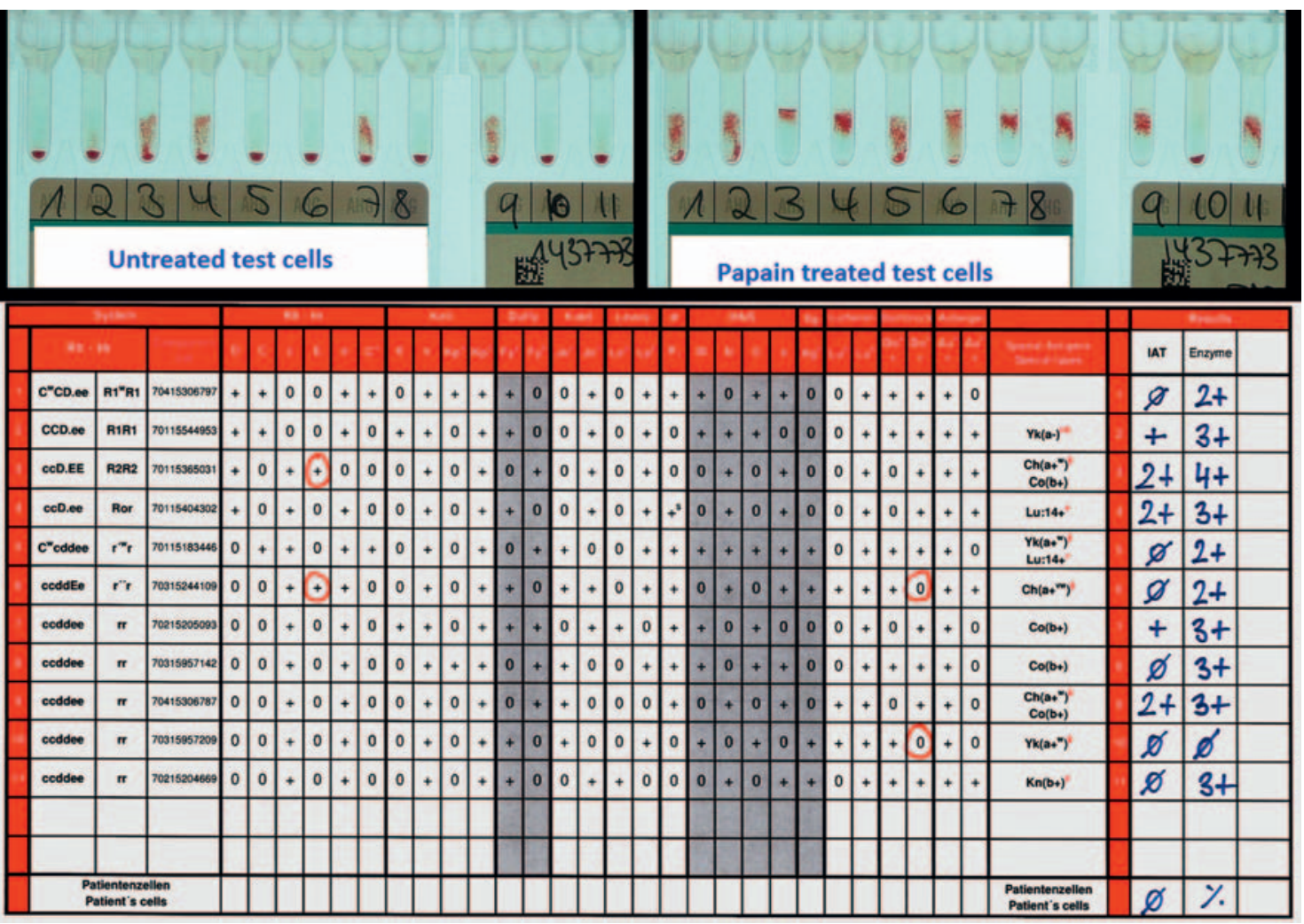


Fig. 3. Antibody identification of patient's plasma contain ing anti- $\mathrm{Au}^{\mathrm{b}}$ and anti$\mathrm{Lu}^{\mathrm{a}}$. The combination of both Lutheran antibodies is almost 'typical'. Slightly enhanced reaction with papain treated cells in AHG.

Fig. 4. Antibody identification: patient's plasma containing anti-MAR (Rh51). Patient: $\mathrm{C}^{\mathrm{w}}$-positive $(\mathrm{D}+$, C+, c-,E-, e+), Genotyping: homozygous $\mathrm{C}^{\mathrm{w}}$-positive. Anti-MAR confirmed using 5 additional homozygous $\mathrm{C}^{\mathrm{w}}$-positive, two sources of $\mathrm{D}$ - and one source of $\mathrm{Rh}_{\text {null }}$ cells.
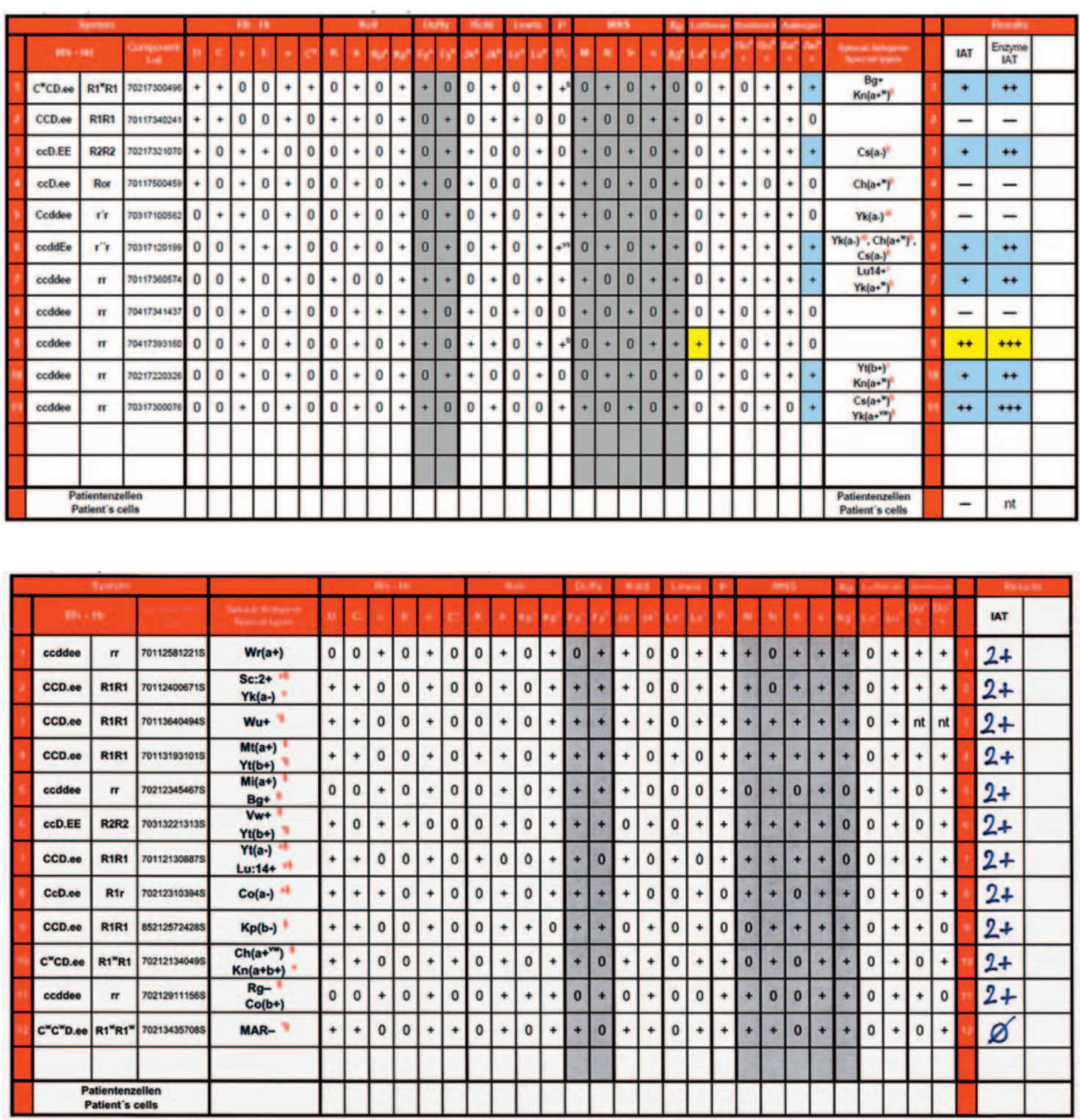

antibodies could be inhibited by the specific recombinant blood group proteins which are commercially available now (Imusyn, Hannover, Germany). In combination with the genotyped reagent RBCs this emerging serological technique is a very helpful tool in antibody diagnostics. It is especially valuable to identify antibodies to high-frequency antigens and to exclude or identify additional antibodies in patients who have antibodies to high-frequency antigens. However the recombinant blood group proteins are available for a limited number of specificities. So far they could not be synthetized for proteins with many transmembrane parts like Rhesus, Diego, or Colton. With few exceptions, the recombinant blood group proteins do not inhibit antibodies to low frequency. We also found single patients with the rare antibody specificities anti-Wu (DI9) and anti$\operatorname{Tar}(\mathrm{RH} 40)$ which is present on red cells with $\mathrm{RhD}^{\mathrm{VII}}$

Anti-MAR-(like) has not been reported in Germany before. Now, we could identify two patients with alloanti-MAR-(like). MAR (RH51) is a high-prevalence Rh antigen which is potentially clinically significant. Only individuals who are combined heterozy- gous for $\mathrm{RhC}^{\mathrm{w}}$ and $\mathrm{C}^{\mathrm{x}}\left(\mathrm{RhC}^{\mathrm{w}} \mathrm{C}^{\mathrm{x}}\right)$ or homozygous for $\mathrm{RhC}^{\mathrm{w}}$ or $\mathrm{RhC}^{\mathrm{x}}$, or who are $\mathrm{Rh}_{\text {null }}$ or $\mathrm{RhD}-$, are MAR-(like)-negative. The original MAR-negative individual $\left(\mathrm{RhC}^{\mathrm{w}} \mathrm{C}^{\mathrm{x}}\right)$ was found in Finland [13]. Our studies revealed that in our donor population in Southwestern Germany only one of 19,000 blood donors is MAR-negative. One of the patients with anti-MAR had an additional anti-S and received compatible blood from MAR-negative and S-negative donors we had found in our previous donor screening.

The clear identification of the antibody specificities is essential to estimate the risk of possible hemolytic transfusion reactions, especially when antibodies to high-frequency antigens are present and serologically incompatible transfusions are necessary. Thus the genotyping of the test RBCs improves the quality of the test reagents and overcomes the limitations of the serological typing caused by shortage and a limited number of licensed test sera. The use of genotyped test cells in antibody identification panels extends the range of detectable antibody specificities, accelerates the antibody identification, and improves the pre-transfusion diagnostics. 


\section{Disclosure Statement}

The employer of the authors, DRK-Blutspendedienst Baden-Württemberg - Hessen, produces commercially available genotyped antibody identification panels.

\section{References}

1 International Society of Blood Transfusion: Red Cell Immunogenetics and Blood Group Terminology www.isbtweb.org/working-parties/red-cell-immunogenetics-and-blood-group-terminology/ (last accessed July $9,2018)$.

2 Storry JR, Castilho L, Chen Q, Daniels G, Denomme G, Flegel WA, Gassner C, de Haas M, Hyland C, Keller M, Lomas-Francis C, Moulds JM, Nogues N, Olsson ML, Peyrard T, van der Schoot CE, Tani Y, Thornton $\mathrm{N}$, Wagner F, Wendel S, Westhoff C, Yahalom V: International society of blood transfusion working party on red cell immunogenetics and terminology: report of the Seoul and London meetings. ISBT Sci Ser 2016;11: 118-122.

3 St-Louis M: Molecular blood grouping of donors. Transf Apheres Sci 2014;50:175-182.

4 Denomme GA, Van Oene M: High-throughput multiplex single-nucleotide polymorphism analysis for red cell and platelet antigen genotypes. Transfusion 2005; 45:660-666.
Wagner FF, Bittner R, Petershofen EK, Doescher A, Müller TH: Cost-efficient sequence-specific primingpolymerase chain reaction screening for blood donors with rare phenotypes. Transfusion 2008;48:1169-1173.

6 Gassner C, Meyer S, Frey BM, Vollmert C: Matrix-assisted laser desorption/ionisation, time-of-flight mass spectrometry-based blood group genotyping-the alternative approach. Transfus Med Rev 2013;27:2-9.

7 Latini FR, Gazito D, Arnoni CP, Muniz JG, de Medeiros Person R, Carvalho FO, Baleotti W Jr, Castilho L, Barreto JA: A new strategy to identify rare blood donors: single polymerase chain reaction multiplex $\mathrm{SNaPshot} \mathrm{reaction} \mathrm{for} \mathrm{detection} \mathrm{of} 16$ blood group alleles. Blood Transfus 2014;12(suppl 1):s256-s263.

8 Avent ND: Large-scale blood group genotyping: clinical implications. Br J Haematol 2009;144:3-13.

9 Rink G, Scharberg EA, Bugert P: PCR with sequencespecific primers for typing of diallelic blood groups. Methods Mol Biol 2015;1310:71-81.
10 O'Shea KP, Oyen R, Sausais L, Stephens VA, Stillwell GF, Bisgard LA, Martin J, Reid ME: A MAR-like antibody in a DCWe/DCWe person. Transfusion 2001;41: 53-55.

11 Mallan MT, Grimm W, Hindley L, Knighton G, Moulds MK, Moulds JJ: The Hall serum: detecting $\mathrm{Kn}^{\mathrm{b}}$, the antithetical allele to $\mathrm{Kn}^{\mathrm{a}}$ (abstract). Transfusion 1980;20:630-631.

12 Westhoff $\mathrm{C}$ et al: Two examples of anti-KCAM, an antibody to an antigen of the Knops system. Tranfusion 2008;48(suppl 2):189A.

13 Sistonen P, Sareneva H, Pirkola A, Eklund J: MAR, a novel high-incidence $\mathrm{Rh}$ antigen revealing the existence of an allelic sub-system including CW (Rh8) and CX (Rh9) with exceptional distribution in the Finnish population. Vox Sang 1994;66:287-292. 